\title{
Electron Diffraction Re-Explained (The Intense Magnetic Fields Interactions within Crystals)
}

\author{
Mahmoud E. Yousif \\ Physics Department - The University of Nairobi P.O.Box 30197 Nairobi-Kenya
}

\begin{abstract}
A model of crystal is suggested, in which atoms are bonded by the Spinning Magnetic Force $\left(F_{S}\right)$ created by the Nucleus Spinning Magnetic Field (NSMF); thus the passing of x-rays through the crystal lattice, having smaller width compared to $x$-ray wavelength, polarized the x-rays, which is part of Electromagnetic Radiation (EM-R) spectrum, by removing it's electric field component and transformed the residual magnetic part into Polarized Wave (PW), or what is known as Conical Diffraction (CD) beam; hence the emergence of $P W$ from crystal is synonymous to the passing of Circular Magnetic Field (CMF) surrounded an electron through such crystal; hence both PW and CMF interacts with the NSMF producing the Glowing Spot-PW (GS$P W)$ for $x$-ray diffraction and Glowing Spot-CMF (GS-CMF) for an electron diffraction, appeared on the monitor screen as dots/rings or reflected as dots patterns; the Kikuchi lines are interpreted as resulted from the interaction of the CMF with the Nucleus Spinning Magnetic Lines of Force (NSMLF), producing the G-NSMLF; thus the interpretation of diffraction as an interaction between wave and matter, is extended to an interaction among intense magnetic fields, and this is the conclusion of this final paper, aimed among others at restoring the common sense in the physical world, removed by pilot wave.
\end{abstract}

Keywords: Circular Magnetic Field; Conical Diffraction Beam; Polarized Wave; Crystal structure; Kikuchi lines; Rings and Patches; Nucleus Spinning Magnetic Field (NSMF); Spinning Magnetic Force (SMFs or $F_{S}$ ); Davisson and Germer experiment; Thomson experiment.

\section{Introduction}

The first part of this work, was "The Double Slit Experiment Re-Explained" [1], in which the diffraction phenomenon was interpreted as resulted from a change in the characteristics of Electromagnetic Radiation (EM-R), when it passed through a single or double narrow openings [2], which represents the foundation for Huygen's principle of diffraction, explained synonymously with semi circle water waves generated in pond [3], which is reinterpreted as a circular wave. When EM-R passed through a small hole/slit/crystal, it is polarized as its electric field component is removed, hence what remained is the magnetic field quantity, where diffraction started by a change in the characteristics of EM-R [1], resulted in what is known as the Conical Diffraction (CD) beam [4], which neither travel with speed of light, nor carrying electric field, and this is designated as the Polarized Wave (PW), the PW is the Circular Magnetic Field (CMF) [5] it's not semicircle, rather it is a full circle, and composed of the magnetic component of the EM-R, it's the CMF, which originated in the integration of both the Circular Magnetic Field (CMF) and the electric field (E-F) during the Flip-Flop $(\boldsymbol{F}-\boldsymbol{F})$ mechanism producing the EM-R [5].

The entre of EM-R in single slit, then changed into PW, produced the same shape of circular wave on the screen [1], while in Young's double slit experiment, the constructive or destructive interference of both PW produced the diffracted patches on the screen; on the other hand, the previously thought phase waves, matter waves [6] or pilot waves [7], in the double slit Electron diffraction experiment [8], is suggested to represents the $\boldsymbol{C M F}$ produced by accelerated electrons, this CMF entre and emerged from both slits/holes in addition to the electron which enters and emerged with the higher magnitude CMF, after which both CMF interfere constructively or destructively [1].

The extension of duality to particles by de Broglie in 1924 [9], was started by Einstein in 1905, when he justified the use of quanta (photon) to knock electron from atom in the photoelectric effect [10], a suggestion endorsed by Compton' experiment in 1922 [11]; the electron diffraction was discovered as a consequence of a deliberate attempt to prove the wave nature of the electron [12], one year after de Broglie suggestion [9], a young graduate (Walter Elsasser), suggested that Davisson and Kunsman's experiment shows evidence of diffraction [12]; in which Davisson and Germer bombarded Nickel crystalline with electrons beam, a diffraction peak wave generated by "electron wave," which was interpreted and derived as the wavelength of Bragg formula resulted in diffraction pattern [13, 14], that was confirmed separately by Thomson and Rupp [6], where the interference patterns by G. P. Thomson was based on passing electrons beam through a thin metal film of gold, aluminum, celluloid and unknown substance $\mathrm{x}$, which resulted in rings patterns formed by cathode rays, similar to $\mathrm{x}$-rays patterns, and thought to agree with de Broglie theory of wave mechanics [15]; although electrons reflected from the nickel crystal in Davisson/Germer experiment, occurred before the detection of diffracted beams [6], which 
questioned how deflected electron could stored reflected phantom wave? And under which of the two states of wave/particle duality does that occurred? Regardless of this, both experiments became decisive in endorsing wave particle duality, making it acceptable, leading to new form of physics, contradicting the common sense and norms of life [16].

In this second part, and after re-explaining the Compton Effect [17], and the double slits experiment [1], a model of crystal is suggested, internally bonded by the Spinning Magnetic Force $\left(S M F c\right.$ or $\left.F_{S}\right)$ produced by the Nucleus Spinning Magnetic Field $(N S M F)$, which bonds atoms, cells and a crystals structure; hence when the crystal is subjected to an X-ray, the EM-R is transformed into Polarized Wave (PW) [1], and since an energetic electron entered into a crystal surrounded by its produced CMF, therefore the $C M F$ produced by an electron and $P W$ resulted from polarized $x$-rays interacted with the crystal's Nucleus Spinning Magnetic Field (NSMF), producing the Glowing Spot-CMF $(G S-C M F)$ and the Glowing Spot-PW $(G S-P W)$, both comprised the $C M F$ shape, and seen as rings pattern on the monitoring screen, or $G S-C M F / G S-P W$ dots reflected with the electron or X-ray from the crystal as in Davisson and Germer experiment [13], while the Kikuchi lines are interpreted as resulted from the interaction of both the Nucleus Spinning Magnetic Lines of Force (NSMLF) with the CMF, thus producing the $G-N S M L F$; all of which showed an interaction between two intense magnetic fields, and this is the conclusion of this final paper, among others aimed at restoring the common sense in the physical world, removed by pilot wave.

As the understanding of the dynamical processes in chemistry, materials science and biology on the micro scale stem almost exclusively from time-resolved spectroscopy [18], therefore knowing the true mechanism of this field, would enrich, unified and expand human understanding and vision, towards much better generations.

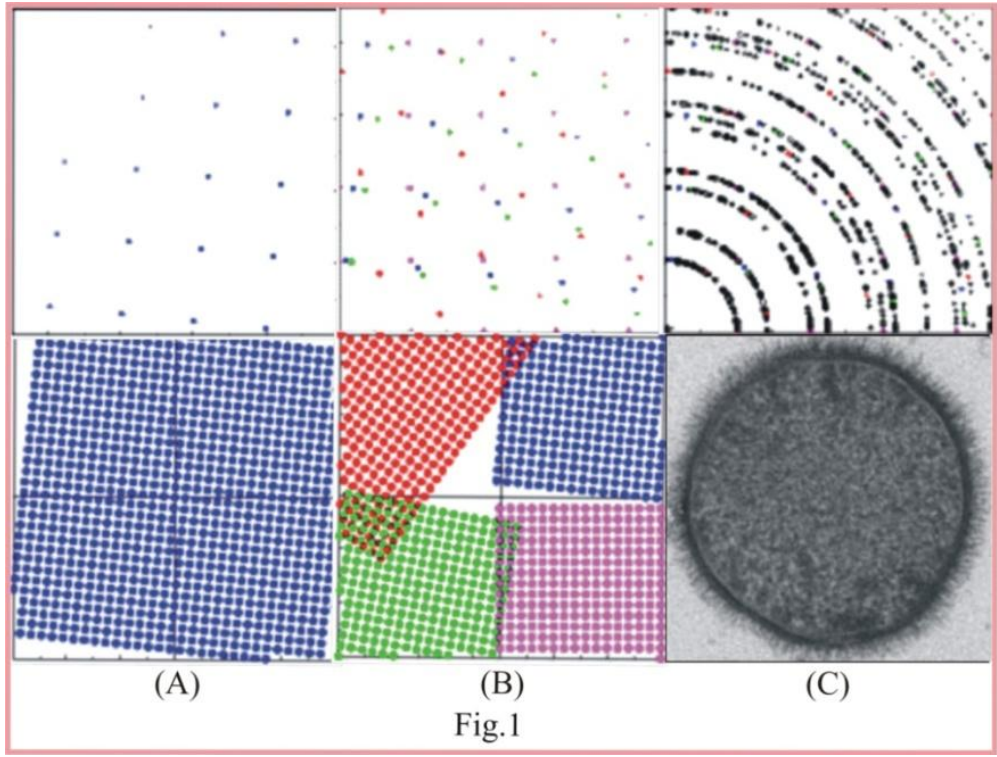

Fig.1. A single crystal diffraction in (A), with a fixed orientation it produced dots pattern on top; the superimposition of four crystals with four different orientations in (B), produced four different groups of dots, each represents specific orientation, while for 40 randomly oriented crystals, powder rings become clearly visible in $(\mathrm{C})$, produced ring pattern [19].

\section{Pilot Wave is the Circular Magnetic Field (CMF)}

A single crystals diffraction is shown in Fig.1-A, with a fixed orientation, the produced dots pattern rotates by the same angle as the crystal; if the number of the crystals are superimposed to four as in Fig.1-B, it gives four different groups of dots, each represents specific orientation, while using 40 randomly oriented crystals powder in Fig.1-C, it produced visible rings [19]; and since it is necessary to rotate the crystal in the $\mathrm{X}$ ray or neutron beam in order to generate the complete diffraction pattern [20], hence the crystal powder gives effects of many layers; but this diffraction is envisaged from the geometrical analogy between light and electron diffraction, based on the smallness of wavelengths of light and electrons compared with the spacing of the diffracted objects [21], as carried by Bragg and based on Huygen's idea [22], although the whole pattern is due to the cathode rays, thought to have been deflected by the film [15]; but G. P. Thomson questioned the nature of this wave? And the relation it has with electrons associated with it [23], and as repeatedly mentioned the only wave produced by an accelerated electron [1], is the Circular Magnetic Field $(C M F)[24,25,26]$, the magnitude of which is given by 


$$
B_{C M F}=\frac{q V_{e}}{r_{m}^{2} c} \quad T
$$

Where, $V_{e}$ is the electron velocity in $\mathrm{m} . \mathrm{s}^{-1}, r_{m}$ is the magnetic radius in $\mathrm{m}, c$ is the speed of light in $\mathrm{m} . \mathrm{s}^{-1}, q$ is charge in Coulomb and $B_{C M F}$ is the $C M F\left(B_{2 e}\right)$ in Tesla, therefore any charged in motion produced Circular Magnetic Field $(C M F)$, the $C M F\left(B_{C M F}\right)$ can be derived in terms of radiation energy $\left(E_{R}\right)$ as [1]

$$
B_{C M F}=\sqrt{\frac{E_{R}^{5} q^{2} 5.12 e+2}{\mathrm{~h}^{4} m c^{6}}} T
$$

Where, $E_{R}$ is the Radiation Energy [1], from Eq. (2), any $C M F\left(B_{C M F}\right)$ can be derived by

$$
B_{C M F}=\sqrt{1.0267123723266052069501087332373 \times 10^{77} E_{R}^{5}}
$$

While, the magnitude of the $C M F-n\left(B_{C M F-n}\right)$ enter and emerge with an electron in any slit is given by [1]

$$
B_{C M F-n}=\sqrt{\frac{2 E_{R} q^{2}}{m r_{m D n}^{4} c^{2}}} \quad T
$$

Where, $r_{m D n}$ is the magnetic radius from electron center to specific $C M F, B_{C M F-n}$ is the $C M F$ intensity from an electron center.

The wavelength due to radiation energy is [1]

$$
\lambda=\frac{C_{B} m c^{7}}{4^{4} 2 E_{R} q^{2}}
$$

Where, $C_{B}$ is the constant of $B_{C M F}$ it is equal to $1.3113864619620884691409896280354 \times 10^{-89} \mathrm{~T}^{2}$. $\mathrm{Hz}^{-5}$ $\left(\mathrm{T}^{2} . \mathrm{s}^{5}\right)$ [1], the wavelength in Eq. (5) is given by

$$
\lambda=\frac{1.98782265 \times 10^{-25}}{E_{R}}
$$

Eq. (6) can reproduce the wavelength versus particle energy for electrons as given by Kittel [27], from which the Radiation Energy $\left(E_{R}\right)$ is [1]

$$
E_{R}=\frac{C_{B} m c^{7}}{2048 q^{2} r_{m D n}}
$$

From Eq. (7), the radius is given by

$$
r_{m D n}=\frac{4.969556625 \times 10^{-26}}{E_{R}}
$$

Therefore, from these equations; an energetic electron, moving through a crystal cells, designated as a direct beam and indicated by 00 [6], is encircled by $C M F$, while an x-ray entering a crystal is transformed into Polarized Wave $(P W)$, and since the $P W$ and the $C M F$ are identical in nature, and produced respectively by both the x-rays and the electrons wave, and the latter could be deflected by electric and magnetic fields [23], similar to the pilot wave [15], and both has similar characteristics [1], therefore both waves interacts with single crystal, several crystals and powder crystals, each in different manner; but how such crystal looks? What force holds atoms in its structure? Answering these questions will help to understand crystals interactions with $C M F \& P W$.

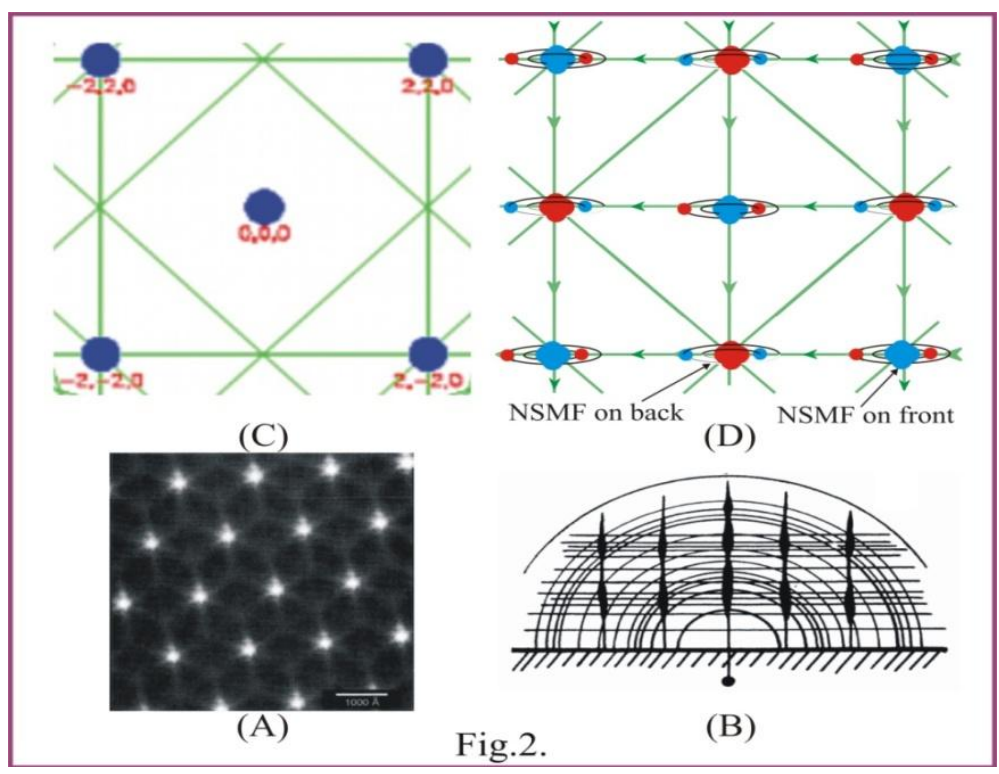


Fig.2. The white diagonal strips of lights connecting the bright spots of the structure shown in (A) [27], is similar in structure to the Kikuchi lines in (C) [30], while (D) shows the front elevation of the proposed crystal shown in Fig.3-A, which is similar in shape to the Kikuchi lines in (C), the blue and red colors shapes are the Nucleus Spinning Magnetic Field (NSMF) of opposite sides. (B) Shows the variable intensities of interaction between Circular Magnetic Fields $(C M F)$ and $N S M F$, the produced puzzle shape; in which the circular spots and the lines become broad bands, the spots are drawn out into lines perpendicular to the shadow edge, the main intensity (or interaction) occurred where the circular bands intersect the horizontal ones [22], or the $C M F$ with SMLF.

\section{Crystal Structure and the Produced Spinning Magnetic Field (SMF)}

The x-ray crystallography work of W. and L. Bragg in 1913 confirmed the microscopic regularity of crystalline matter [28], although the spatial characteristics of crystal lattices, was investigated and became known, but the forces holding these units in position are still not completely understood, [6], it is stated that, in inorganic crystals, the dominant bonding forces are ionic or heteropolar bonds and covalent or homopolar bonds, with lesser contributions from van der Waals and hydrogen bonds, and the ionic bond is non-directional, this is shares with the metallic bond which is the dominant cohesive force in metals with high conductivity [21], while the attractive electrostatic interaction between electrons and the nuclei is entirely thought responsible for the cohesion of solids, and magnetic forces is thought to have weak effect [27], but to what extend does that have?

Though results of our investigations are contrary to that, showing magnetism playing great roles in many aspects, particularly in holding electron to the nucleus [29], hence magnetic force is suggested to bond crystal atoms.

The crystal is a homogeneous solid that possess long-range, three dimensions internal order, and the unit cell is the smallest unit of the structure (or lattice) that can be indefinitely repeated to generate the whole structure (lattice) [30], and a single crystal is composed of unit cells of the Bravais lattices, represents a structural unit or building block that can describe the crystal structure [21], [31], the structure repeats itself periodically in each of the three dimensions [8], thou the white diagonal strips of lights seen to connect the bright spots within the structure shown in Fig.2-A [27], is similar to the enlarged Kikuchi lines shown in Fig.2-C [30], and thought to be formed in diffraction patterns by diffusely scattered electrons [32], the Kikuchi Lines appear in pairs, being parallel to one another, the black lines (refers to difference of intensity), often passes through a spots, and is then perpendicular to the line joining the spot to the origin [8], it moves during tilt as if they are affixed to the bottom of the crystal [30], the Kikuchi patterns have been used to determine the orientation [33], as they are fixed within the crystal [34] so far the part played by electron forward-focusing effect in Kikuchi band formation remained undisclosed [35], although Kikuch interpreted these lines as the amount of diffuse scattering of the entering beam, reflected from various sets of planes with which they make the Bragg angle, but it doesn't account for the production of the diffuse scattering which is a loophole in this theory [8]; however the Kikuchi lines which are presented in form of stereographically projection of two dimensional scattered electron intensity map in polar and azimuthally takeoff angles [35], is perceived as if representing what a fisheye lenses camera can shoot while inside such crystals, and since the intersection of Kikuchi lines near a spot, caused partial suppression to the spot, suggesting an interference between the rays forming the spots and those forming the line [8], thus giving evidence for a links between the spots and Kikuchi lines, hence the spots are interpreted as Nucleus Spinning Magnetic Field (NSMF) [36] and Kikuchi lines as Spinning Magnetic Lines of Forces (SMLF), similar to sunspots magnetic lines of force seen in extreme ultraviolet wavelength [37], therefore both NSMF and SMLF are suggested to form a mechanism bonding atoms to form crystals cells, in which the Kikuchi bands mark orientation space with well-defined intersections [32], known also as Kikuchi poles [38], or zones as well as paths connecting one intersection to the next [32], they are herby suggested to connect each NSMF to another, therefore such unit cell of Silver crystal with Faced-Centered Cubic (FCC) in which atoms are found at the centers of the six faces of the unit cell as well as at the eight corners, as in $\mathrm{Al}, \mathrm{Cu}, \mathrm{Ag}, \mathrm{Pt}$ [6], others such Elements with Monatomic FCC Crystal Structure are: Ar, Au, Ca, Ce, Ir, Kr, La, Ne, Ni, Pb, Pd, Pr, Xe and Yb [28], and since parallelepipeds, which is the unit cell, may be formed in an infinite number of ways, as they may be bonded by any three sets of parallel lines through the lattice points, shown in Fig.3-B\&C by the three orange heads, while in each case the volume of the cell is the same [8], therefore this is suggested to composed of structure shown in Fig.3-A, the suggested structure is similar to the two dimension lattices of the unit cell of the square $\mathrm{p}$ lattice $(\mathrm{p} 4 \mathrm{~mm})$ shape [21], the faced-centered cubic Bravais lattice is related to the simple cubic lattice, while addition structure is shown by the four directions in Fig.3-B [28], representing the atom as shown by the basic unit structure in Fig.3-C, while part of an enlarged structure of the front elevation of Fig.3-A, is shown in Fig.2-D, with great similarities with the adjacent Kikuchi lines in Fig.2-C [30]; while in the model shown in Fig.3-A, each atom produced Nucleus Spinning Magnetic Field (NSMF) [36], similar to proton as nucleus in hydrogen atom, that spins to produce Spinning Magnetic Field (SMF or $B_{1 U}$ ) [36], which when interacted with electron's $C M F$ produced the Magnetic Force $\left(F_{m}\right)$, subsequently at specific radial distance the Magnetic Force 
$\left(F_{m}\right)$ equalized the Electrostatic Force $\left(F_{e}\right)$, and both forces balanced the Centripetal Force $\left(F_{c}\right)$ to form the hydrogen atom [29], and since hydrogen atoms under certain conditions attracted by rather strong forces to two atoms, thus formed triple hydrogen bonds of difluoride ion $\left(\mathrm{HF}_{2}\right)$ [27] therefore the nucleus of an atom shown in Fig.3-C, represents the building block for the formation of crystal structure, with arrows in orange and green gives field direction, hence each atom in the crystal unit cell [28, 31], shown in Fig.3-A, composed of Silver Nucleus Spinning Magnetic Field $\left(B_{1 U A g}\right)$, derived from the Total Nucleus spinning magnetic field (NSMF) produced by a protons and neutrons given by [36]

$$
B_{T N E}=A B_{N S M F} r_{r}^{2} \quad \text { T. } m^{2}
$$

Where, $\mathrm{A}$ is the number of nucleon $(\mathrm{Z}+\mathrm{N}), B_{N S M F}$ is nucleus $S M F, r_{r}$ is distance from nucleus surface along the magnetic field, $B_{T N E}$ is the total magnetic field produced above each pole of an element $E$, therefore the total spinning magnetic field (SMF or $B_{T N E}$ ) of nucleus can be derived from the following equations [36], and given by

$$
B_{T N E}=A \frac{\mu_{0} q}{4 \pi} \sqrt{\frac{2 q^{2} r_{o}}{\varepsilon_{0}\left(f_{P S}+f_{N S}\right)\left(m_{P}+m_{n}\right)\left(r_{P}^{2}+r_{n}^{2}\right)}} T \cdot m^{2}
$$

Where, $r_{o}$ is the radial distance from nucleus surface to a point at which $B_{N S M F}$ is produced $\left(r_{0}=0.468 \mathrm{fm}\right)$. Since most parameters in Eq. (10) are constant, hence the $B_{N S M F}$ is given by

$$
B_{T N E}=A 1.7031777063137652486648653357669 \times 10-20 \text { T. } m^{2}
$$

From Eq.(11), related to Eq. (9), the nucleus spinning magnetic field (NSMF or $B_{N u S M F}$ ) is

$$
B_{N S M F}=\frac{B_{T N E}}{r_{r}^{2}} \quad T
$$

\begin{tabular}{|c|c|c|c|}
\hline $\begin{array}{c}E \\
\mathrm{kV}\end{array}$ & $\begin{array}{c}V_{e} \times 10^{8} \\
\mathrm{~m} / \mathrm{s}\end{array}$ & $\begin{array}{c}\operatorname{CMF}\left(B_{C M F}\right) \\
\mathrm{T}\end{array}$ & $\begin{array}{l}r_{m} \\
m\end{array}$ \\
\hline 100 & 1.64 & $\begin{array}{c}1.0411201542542049102350072469 \\
336 \times 10^{4}\end{array}$ & $\begin{array}{c}3.1017519296693581352820664364287 \\
\times 10^{-12}\end{array}$ \\
\hline 200 & 2.09 & $\begin{array}{c}5.8894649688250614173649365167 \\
233 \times 10^{4}\end{array}$ & $\begin{array}{c}1.5508759648346790676410332182144 \\
\times 10^{-12}\end{array}$ \\
\hline 300 & 2.33 & $\begin{array}{c}1.6229457035570067421544247612 \\
001 \times 10^{5}\end{array}$ & $\begin{array}{c}1.0339173098897860450940221454762 \\
\times 10^{-12}\end{array}$ \\
\hline 400 & 2.48 & $\begin{array}{c}3.3315844936134557127520231901 \\
876 \times 10^{5}\end{array}$ & $\begin{array}{c}7.7543798241733953382051660910718 \\
\times 10^{-13}\end{array}$ \\
\hline 1000 & 2.82 & $\begin{array}{c}3.2923110053491292648023767947 \\
019 \times 10^{6}\end{array}$ & $\begin{array}{c}3.1017519296693581352820664364287 \\
\times 10^{-13} \\
\end{array}$ \\
\hline
\end{tabular}

Where, $B_{T N E}$ is the total magnetic field produced above each pole in Fig.3-A, and $B_{N S M F}$ is nucleus $S M F$ in Tesla.

Table1. Electrons properties of velocity $\left(V_{e}\right)$ and Circular Magnetic Field $\left(C M F B_{C M F}\right)$ depends on the acceleration potential (E), the $C M F$ is derived using Eq. (3), and $r_{m}$ using Eq. (8).

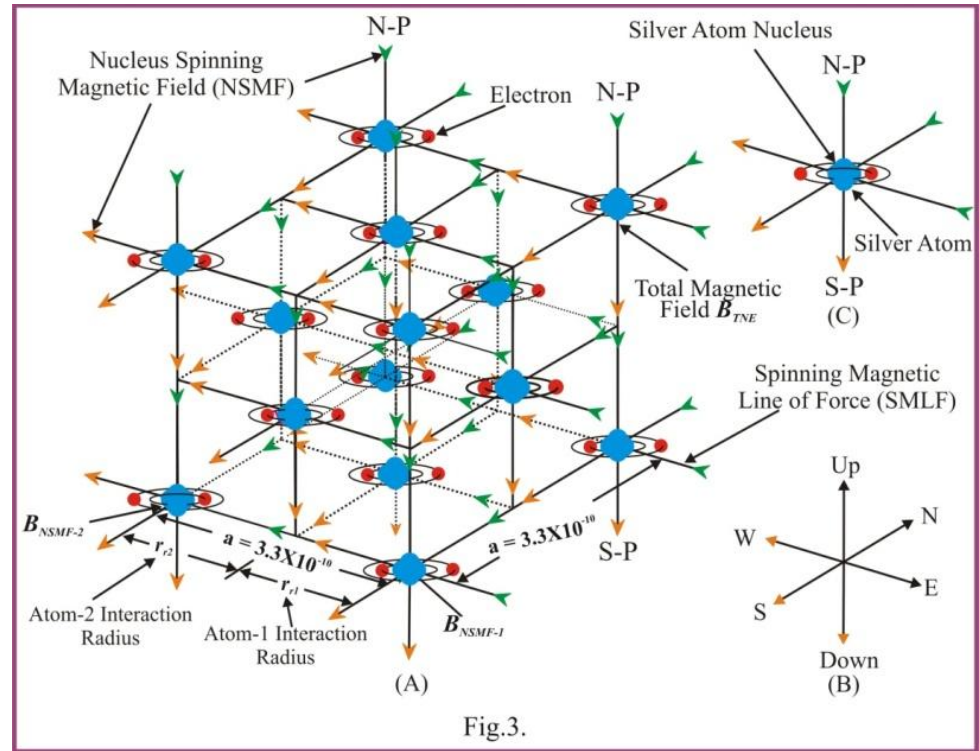

Fig.3. Stick and Ball Model of a single Aluminum Lattice Faced-centered cubic (FCC) structure and bonds in (A), it composed of fourteen atoms [31], each in blue, suggested to bond with each other through the Spinning Magnetic Line of Force (SMLF) [36] stick with orange and green colors heads, the SMLF are produced by the 
Nucleus Spinning Magnetic Field (NSMF), resulted in Spinning Magnetic Force ( $S M F s$ or $F_{C}$ ), while (B) shows the six directions of each atomic SMF, and (C) is the unit atom with $S M F$, representing the building block for crystal structure formation.

\section{Inter-Crystal Bonding Force}

Since Thomson experiment showed the presence of the central spot when the film is not used, and the central spot and rings are deflected together, and they are due to cathode rays of significantly the same velocity [15], and since electrons interacted strongly with the sample $10^{4}$ times stronger than x-rays [39], hence the diffracted beams are also strong and can, in turn act as incident beam [40], therefore these characteristics are greatly related to the $C M F$ which surrounds an energetic electrons as given by Eq. (1) and shown in Table. 1; while the appearance of four spots on the circumference of the diffracted rings, causing non-uniform intensity, mentioned first by Thomson [15], is a phenomenon required special attention; the four spots in the diffraction patterns of deposited silver ( $\mathrm{Ag}$ ) thin films is shown in Fig.4-I, in which an Ag thin films is deposited on the $\mathrm{NaCl}$ crystals at various temperatures, they are divided into five stages of epitaxy (A1.1); in the first stage at Fig.4-I-a, Debye rings fully appeared when the substrate temperature was normal, but when the temperature is raised by $\mathrm{R}=25 \%, 50 \%, 75 \%$ and $100 \%$ in (b), (c), (d) and (e) respectively, the Debye rings gradually disappeared with an increase in temperature, till it vanished completely at higher temperature in Fig.4-I-e [41]. The complete disappearance of rings in Fig.4-I-e, with the appearance of equidistance set of lights, suggested to represents the atoms; and since the five figures of Fig.4-I-(a-b-c-d-e) supposed to have equal dimensions, therefore the circumference of the central light in Fig.4-I-e is greatly reduced, meaning the intensity is greatly reduced; and this also attained experimentally, when an increase in the temperature of the crystal lead to decreases in the intensity of the Bragg-reflected beams [27], while rubbing polycrystalline specimens altered the diffraction pattern completely, and rings almost disappeared, leaving a central row of diffused spots [8], as rubs' generate heat; thus the elimination of the rings and reduction intensity of the central beam are linked to an increased of heat, therefore this can be explained based on the crystal cell mechanism showed in Fig.3-A, wherein each atom in the cell generates Nucleus Spinning Magnetic Field (NSMF), and since the magnitudes of magnetic field decreased with an increased heat [42], and the decreases of line width for nuclei caused atoms to jump between crystals, while an increase in temperature greatly decreases the average time $\tau$ an atom remains in one site [27], which means a decrease in the produced NSMF, hence these characteristics, are related to the magnitude of interaction between the electron' CMF and atom' NSMF on each unit cell; therefore it is suggested that each atom in the unit cell shown in Fig.3-A constitute Nucleus Spinning Magnetic Field (NSMF), the magnitude of which given by Eq. (12), and that, as shown in Fig.3-A, each atom in the crystal unit cell is bonded with each other, and with other crystals unit cells through the Spinning Magnetic Force $\left(F_{S}\right)$ [29] created by two NSMF, forming structural material shown in Fig4-II, the field direction for each NSMF is shown as either emerging from an atom as south-field or protrude into an atom as north-field as shown by the green and orange heads in Fig.3-A, each atom is bonded by these strong NSMF lines of force, their lines are similar to Kikuchi lines shown in Fig.2-C, which is compared to the side elevation of silver crystal cell model in Fig.2-D, therefore the force between two atoms in this model, shown in Fig.3-A is given by

$$
F_{A S}=B_{N S M F 1} B_{N S M F 2} r_{r 1} r_{r 2} c \sin \theta
$$

Where as shown in Fig-3-A, $B_{N S M F 1}$ is the NSMF of the first atom, $B_{N S M F 2}$ is NSMF of the second atom, $r_{r 1}$ is the first atomic radius, $r_{r 2}$ is the second atomic radius, and $F_{A S}$ is the Atom Spinning Magnetic Force in Newton [29]; organizing Eq. (13), this force can be given by

$$
F_{A S}=\left(\frac{B_{T N E-1}}{r_{r}^{2}}\right)\left(\frac{B_{T N E-2}}{r_{r}^{2}}\right) r_{r 1} r_{r 2} c \sin \theta
$$

Since atom of specific element produced equal magnitude of NSMF, and the distance $r_{r}$ is the same, therefore Eq. (14) can be reduced to

$$
F_{A S}=\left(\frac{B_{T N E}^{2}}{r_{r}^{2}}\right) c
$$

\section{The Fisheye Circular Magnetic Field (FSH-CMF)}

The Bragg law resulted from the observation that "the Laue spots on the $x$-ray photograph of zinc blended, are elliptical, not circular in shape, with the short axes lying in radial directions" [21], but the central spot on Fig.4-I-e is circular in shape, in addition to that, Thomson based his experiment on the assumption that by sending electron beam through a film of randomly arranged minute crystals, and if that resulted in pattern identical to the one of Hull-Debye-Scherrer who used x-rays of the same wave-length, then de Broglie suggestion was correct [15], but in the relaxation illustration shown in Fig.2-B, the circular spots and the lines become broad bands, the spots are drawn out into lines perpendicular to the shadow edge, the main intensity occurred where the circular bands intersect the horizontal ones [22], but the shapes of these circular bands are identical to the $C M F$, while the NSMF are situated along the horizontal lines; thus the intersections in Fig.2-B, 
are interpreted as points of interactions between the two strong fields of the CMF and the NSMF; hence the discrepancies between these diagrams and diffraction interpretation showed the existence of different mechanism, but as given by Eq. (1) and Fig.4-II, electrons produced wave of $C M F$ upon which $E M-R$ is based [5], and since pattern of concentric rings on fluorescent screen are centered around the in-diffracted electron beam [43], showing the concurrent existence of both the electron and the wave, hence when electron beam is accelerated through the specimen shown in Fig.5-A, and since the forwards and backwards movement of photographic plate, shows the spots are formed by rectilinear pencils spreading in all directions from the crystal [22], and as each electron is encircled with CMF, the magnitude of which is proportional to the accelerating potential and inversely with the radial distance as given by Eq. (1), and Eq. (4), and in Table.1; and since electron beam in the transmission electron microscopy (TEM) is make to travels through the sample then focused on a detector plate [27], which is the same principle used by Thomson [15], therefore when such an electron entered an aluminium specimen, as shown in Fig.4-II, it's encircled by the $C M F$ with intense magnitude nearer electron and weaker farther along the radial distance from it as given in Table.1; and since all results obtained with films of aluminum, gold and celluloid produced series of concentric rings around the spot made by the undeflected beam [15]; and the experimental works showed the rings radius decreased with an increase in the accelerating potential [44], as derived by Eq. (1), Eq. (4) and Eq. (7), and given in Table 1; and since the diffraction spots blink on and off as one tilts the crystal, while Kikuchi bands mark orientation space with welldefined intersections (called zones or poles) as well as paths connecting one intersection to the next [32], and since the amount of scattering by atoms of heavy elements ( $\mathrm{Au}, \mathrm{Ag}$ ) is so large that the Laue theory can hardly expected to hold [45], and since the diffraction approach is not correct in a physical sense, but only correct in a geometrical sense [21], and the rings and the bright spots in Fig.4-II are showed to relate to a form of magnetic interaction between $C M F$ and $N S M F$, and since the passage of $\delta-B_{C M F}$ of electromagnetic radiation $(E M-R)$ through a slit or aperture transformed the radiation into $P W$, which is alike to $B_{C M F}[1]$, therefore an $\mathrm{x}$-rays entering the much smaller width of crystal cell specimen is transformed into $P W$, hence in the following discussion, $C M F$ produced by electron and $P W$ resulted from polarized x-rays are similar in nature, content and identical [1], therefore both electron's $C M F$ and x-ray's $P W$ interacts similarly with NSMF (only differ in intensity); and since in microscopic-level, the probe electrons and radiation interacted with the object under study to create an image [27], and the dynamic range in the Kikuchi images could be so large that only portions of the film are not overexposed [32]; and since the white Kikuchi line must always be nearer the central spot than the black line [8], indicating strong relation with the nucleus; and remembering that, objects illumination in electron microscope are thought to be either self-luminous, or illuminated by an external source [46]; in the later, it's claimed every point supposed to emit radiation independently of all others, and, as the phases of the light emitted by them are distributed at random, the resulted intensities in the image will be the same as if only one point emitted light at a time, and the elementary intensity components were summed up [46], this is an inconceivable interpretation; in addition to the fact that, the x-ray beam in the x-ray photograph of deoxyribonucleic acid (DNA) is normal to the fiber and the diffraction pattern is characterized by four lozenges or diamond-shapes outlined by fuzzy diffraction haloes and separated by two rows or arms of spots radiating outwards from the centre [21], all these self-luminous systems raised several questions about its mechanism and energy source, and since diffraction is loosely defined as the flaring of light as it emerges from a narrow slit [47], therefore it is suggested that, in the test showed in Fig.5-A, when electrons flow through the crystal cells, the produced $C M F$ travelled through the cells and interacted with each cells' $N S M F$, resulted in the production of Glowing Spot CMF $(G S-C M F)$ for electron' CMF and Glowing Spot PW $(G S-P W)$ for an x-ray's wave, therefore specimen number one in Fig.5-A produced the GS-CMF for each unit cell, they travelled directly with electron, and enlarged on the monitor screen as shown in Fig.5-C, which showed traces of the CMF in addition to the resulted $G S-C M F$, hence the screen reflects the amplification of what the $C M F$ detected, captured or interacted with at the specimen holder (flaring of light [47]); this explained the improperly interpreted single crystal diffraction [8], shown in Fig.1-A. 


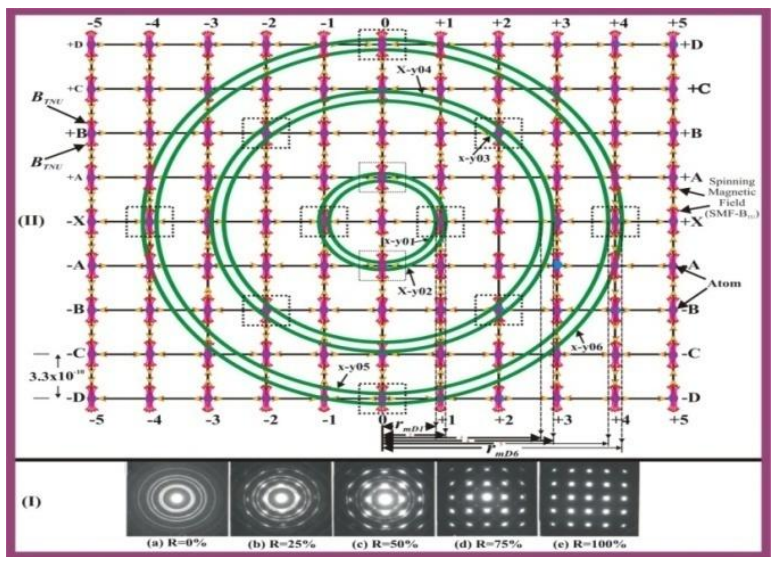

Fig.4. In (I) changes in pattern by variation in temperature; divided into five stages of epitaxy, from law as $0 \%$ at (a), to $\mathrm{R}=25 \%, 50 \%, 75 \%$ and $100 \%$ in (b), (c), (d) and (e) respectively [41], this decreased the magnitudes of Nucleus Spinning Magnetic Field (NSMF), thus reducing interaction intensity between Circular Magnetic Field $(C M F)$ and Nucleus Spinning Magnetic Field $(N S M F)$, while (II) shows the points at which $C M F$ interacted with the $N S M F$, creating the Glowing Spots-CMF $(G S-C M F)$ and ring patterns; (II) also shows the interaction Magnetic Radius $\left(r_{m D n}\right)$.

If a sample of 40 randomly oriented crystals powder is inserted in the holder, as shown in Fig.5-B, the electron produced $C M F$ travelled with it through the specimen, each $C M F$ interacted with each of the cells' $N S M F$ on its path producing $G S-C M F$, thus traces of the $C M F$ are glows, and appeared on the monitor screen in form of dots of rings as shown in Fig.5-D, the formation of these rings are shown in the cross-section elevation of Fig.4-II, depicting the silver atom diffraction shown in Fig.4-I-c; therefore each radial intensity $C M F$ or $B_{C M F}$ shown in Fig.4-II and given by Eq. (4) interacted with four NSMF transverse its path, the intense magnitude of the $C M F$ near the electron interacts intensely with the first four groups of atoms' NSMF shown in Fig.4-II, the next four groups are twisted $45^{\circ}$, due to the arranged shape of the crystals and related NSMF, the third group is also twisted $45^{\circ}$ from the previous; similar to the diffraction photo shown in Fig.4-I-b\&c; the total number of rings shown in Fig.4-II, due to these interactions are six, depicting the main silver pattern rings in Fig.4-I-a, while the rings are completely disappeared in Fig.4-I-e, therefore when NSMF is weak, it is imprinted on the $C M F$, without forming rings, but before weakened, as the photograph in Fig.4-I-b\&c shows, each four groups of atoms are encircled with two rings; hence the interaction of strong fields of $C M F$ by weak NSMF resulted in the glowing of two strips of $C M F$ surrounding the $N S M F$ as shown in Fig.4-I\&II, and the usage of powder as shown in Fig.1-C is just to superposed the pattern produced by an individual crystals with random orientations [19], where it enhanced the rings due to the randomness and strong NSMF emerged from each cell; in addition to the availability of such four strong NSMF emerged from each cell; while many cells layers caused such effects, as depicted and shown in Fig.4-II, but since the intense interaction between CMF-NSMF eliminates the patches appearance of the NSMF, as in Fig.4-I-a; and since the sequence of ring spacing is a characteristic of the FCC structure [21], hence the axis of interaction between each $C M F$ and related four $N S M F$, is derived from the central electron beams outwards, as shown in Fig.4-II, and given in Table.2, as related to the four NSMF magnitude, they are:

$(\mathrm{xy}-01)+\mathrm{A}+0,+\mathrm{X}+1,-\mathrm{A} 0,-\mathrm{X}-1$

$(\mathrm{xy}-02)+\mathrm{A}+0,+\mathrm{X}+1,-\mathrm{A} 0,-\mathrm{X}-1$;

$(\mathrm{xy}-03)+\mathrm{B}+2,-\mathrm{B}+2,-\mathrm{B}-2,+\mathrm{B}-2$;

$(\mathrm{xy}-04)+\mathrm{B}+2,-\mathrm{B}+2,-\mathrm{B}-2,+\mathrm{B}-2$

$(\mathrm{xy}-05)+\mathrm{D} 0,+\mathrm{X}+4,-\mathrm{D} 0,-\mathrm{X}-4$;

$(\mathrm{xy}-06)+\mathrm{D} 0,+\mathrm{X}+4,-\mathrm{D} 0,-\mathrm{X}-4$;

These interactions resulted in the formation of the ring patterns shown in Fig.4-II, and if the thickness of

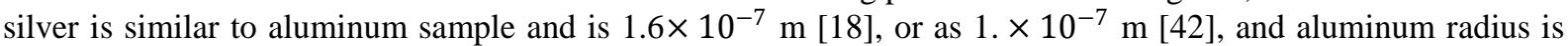
$1.48 \AA$ [48], and distance $a=4.04 \times 10^{-10} \mathrm{~m}$ [40] therefore, the layers (or cells number) for a sample range between 247-396 layers (cells), for a silver, and since $\mathrm{a}=3.3 \times 10^{-10} \mathrm{~m}$ [49], hence the numbers of cells' layers penetrated by electron' CMF are 500; transverse, before an electron emerged from the sample, and since the circumference of rings are uniformly round in some cases; while the intensity is more or less concentrated in a series of spots on the circumference in other cases [15], therefore this is the interaction between $C M F$ and NSMF with various over exposure results [44] causing this, while the $C M F$ transverse the 247-396 or 500 layers of crystal cells. 


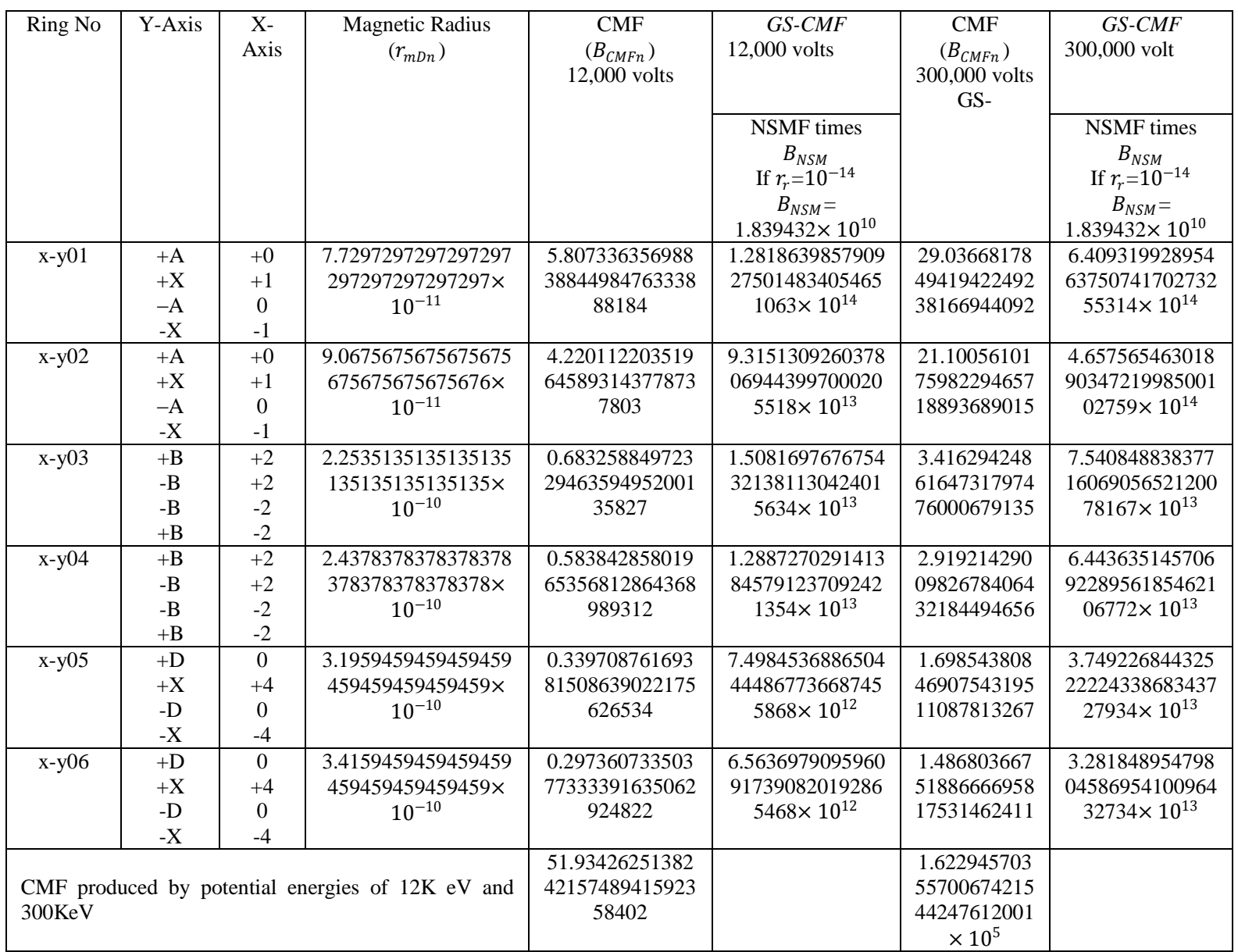

Table.2. The interaction of Circular Magnetic Field $(C M F)$ or Polarized Wave $(P W)$ with multiple Silver Atom Nucleus Spinning Magnetic Field (SA-NSMF), producing six Glowing Spot-CMF (GS-CMF), or Glowing Spot-PW (GS-PW) rings shown in Fig.4-II; each ring is numbered from the center outwards preceded by $\mathrm{x}$-y coordinates, as given on the first Colum, then the Y-coordinate and X-coordinate for each point, the Magnetic Radius $\left(r_{m D}\right)$ derived from the relative length $-\mathrm{a}$ - in Fig.4-II for silver its $1.65 \times 10^{-10} \mathrm{~m}$ [49] $r_{m D}$ is derived from the figure, then the first CMF $\left(B_{C M F}\right)$ produced by potential of $12 \mathrm{KeV}$ using Eq. (4), then the first GS-CMF using Eq. (15), then second CMF $\left(B_{C M F}\right)$ produced by potential of $300 \mathrm{KeV}$ using Eq. (4), then the second GS-CMF using Eq. (15), and the NSMF using Eq. (12), $n=4$ and $l=300$. 


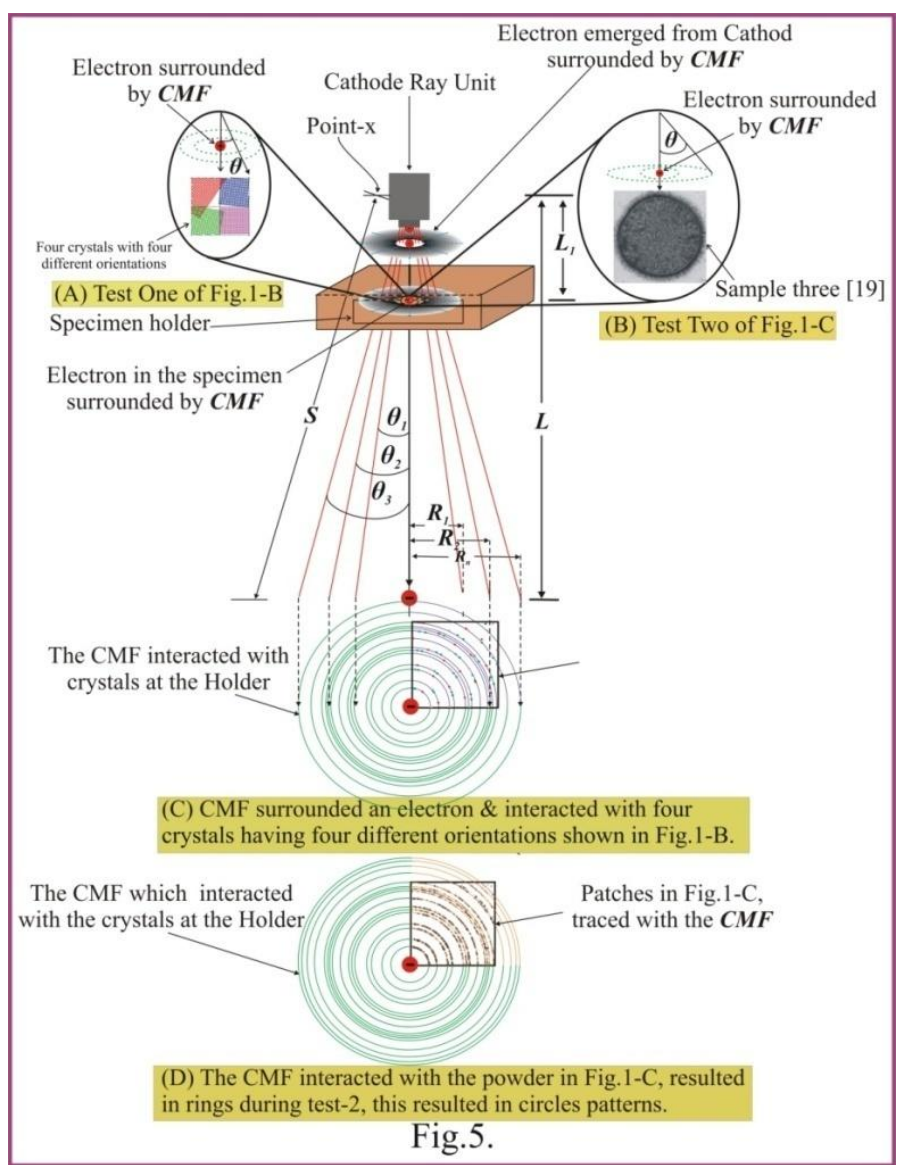

Fig.5. The Circular Magnetic Field $(C M F)$ while enlarging and moving with the electron from the cathode ray to the screen, like a rectilinear pencils spreading in all directions from the crystal [22], (A) shows the superimposition of four crystals with four different orientations given in Fig.1-B, while (B) contains 40 randomly oriented crystals, powder rings as shown in Fig.1-C. An accelerated electron in (C) surrounded by $C M F$, entered the test holder, interacted with the Nucleus Spinning Magnetic Field (NSMF) of sample (A) resulted in Glowing Spot-CMF (GS-CMF) dots in $C M F$ forms imprinted on the monitor screen at (C), using sample in (B), the interaction resulted in clear trace of rings pattern shown in (D). The figure also shows the distance $(L)$ between the cathode and the screen, the distance $\left(L_{l}\right)$ between the cathode and the specimen, the ring radius $\left(R_{n}\right)$ and the angle $\theta$ between electron trajectory and the $C M F$ on which $G S-C M F$ is imprinted, or the ring radius.

The interaction between the $C M F\left(B_{C M F}\right)$ and the $N S M F\left(B_{N S M F}\right)$, resulted in data given in Table.2, as derived from Fig.4-II, and given by

$$
G S-C M F(P W)=n l\left(B_{C M F n} \times B_{N S M F}\right)
$$

Where, $B_{C M F n}$ is the $C M F$ given by Eq. (2), $B_{N S M F}$ is the magnitude of Nucleus Spinning Magnetic Field given by Eq. (12), $\mathrm{n}$ is the number of $N S M F$ interacted with each $C M F$ (four), $l$ is the number of crystal cells layers transverse by the $C M F$ before appears on the monitoring screen and $G S-C M F$ is Glowing $C M F$; but since $B_{C M F}$ is given by Eq. (4) and NSMF is given by Eq. (12), therefore substituting both in Eq. (16), hence GS-CMF is given by

$$
G S-C M F(P W)=n l\left\{\left(\frac{B_{T N E}}{A r_{r}^{2}}\right) \times\left(\sqrt{\frac{2 E q^{2}}{m r_{m D n}^{4} c^{2}}}\right)\right\}
$$

Although silver atom radius was given as $1.52 \times 10^{-10} \mathrm{~m}$ [27], the current radius is $1.65 \times 10^{-10} \mathrm{~m}$ [49], hence using this as silver atom radius in Fig.4-II, this gives a $=3.3 \times 10^{-10} \mathrm{~m}$ for distance $-\mathrm{a}$ - from which the relative Magnetic Radius $\left(r_{m D n}\right)$ for each of the six rings are measured and given in Table.2; then using two acceleration potential the $12 \mathrm{keV}$ used by Thomson [15], and $300 \mathrm{keV}$ typically used in electron microscopes [40], to derive the $C M F\left(B_{C M W}\right)$ for each potential using Eq. (4), while the magnitude of NSMF is derived for silver atom using Eq. (12), then the GS-CMF (GS-PW) for both are derived using Eq. (16), as given in Table 2.

The acceleration potential (E) of $12 \mathrm{keV}$ is used in Table.3, in addition to the $300 \mathrm{keV}$, thought to correspond to the wavelengths of $0.020 \AA$ [40], in addition to 935.3 volts to derive de Broglie wavelength and compared it with 
the relative Magnetic Radius $\left(r_{m D n}\right)$ derived from Fig.4-II, and the wavelength of the first ring which is $\lambda=$ $r_{m D 1} \times 4$.

\begin{tabular}{|c|c|c|c|c|}
\hline $\begin{array}{c}\text { Ring One Magnetic } \\
\text { Radius }\left(r_{m D 1}\right)\end{array}$ & $\begin{array}{c}\text { Ring one } \lambda \\
\left(r_{m D 1} \times 4\right) \\
\text { For 935.3 Volts }\end{array}$ & $\begin{array}{c}\text { de Broglie } \\
\left(\lambda_{d}\right)\end{array}$ & $\begin{array}{c}\text { de Broglie } \\
\left(\lambda_{d}\right) \\
12 \mathrm{KeV}\end{array}$ & $\begin{array}{c}\text { de Broglie } \\
\left(\lambda_{d}\right) \\
300 \mathrm{KeV}\end{array}$ \\
\hline 7.7297297297297297297 & 3.091891891891891891 & 4.010199542287126776 & 1.119568903458237982 & 2.239137806916475964 \\
$297297297297 \times 10^{-11}$ & $8918918918919 \times 10^{-10}$ & $6498554576561 \times 10^{-11}$ & $2641426924593 \times 10^{-11}$ & $5282853849187 \times 10^{-12}$ \\
\hline
\end{tabular}

Table.3. The comparison between the Magnetic Radius $\left(r_{m D 1}\right)$ of the firs ring, the equivalent wavelength for the first ring is $\lambda=r_{m D 1} \times 4$, and de Broglei wavelength using Eq. (18) with potential $935.3 \mathrm{~V}$, $12 \mathrm{KV}$ and $100 \mathrm{KV}$ respectively, the magnetic radius as quarter of the wavelength, shows why it took so long to be defined.

\section{Davisson and Germer Experiment}

It was stated by Harnwell that, "Davisson and Germer reflected electrons from a nickel crystal and detected the diffracted beams electrically" [6], but which reflection occurred first? The electron or the wave or both the electron and the phantom wave deflected simultaneously? If Harnwell statement is correct, the diffraction is either formed before or after the reflection, although for Davisson and Germer, both the reflected incident of wave and electron occurred concurrently [13], which means, the wave is part of the electron in both journeys, contradicting the either wave/particle duality perceived by the electron; but as been raised, what is the wave pulses reflected by crystal's internal components, is it secondary wave as suggested by Bragg based on Huygen's idea [22]? And how does it related to X-ray? Though the reflection of electron wave from a crystal is not analogy to the X-ray reflection [8], regardless of the resulted similarity; hence what differ them?

Great similarities thought to exist between the scattering of electrons by the crystal and the scattering of waves by three and two dimensional gratings [13], and since the passing of electromagnetic radiation $(E M-R)$ through a relatively small slit, will transformed it into Polarized Wave $(P W)[1]$, therefore the passing of the $\mathrm{x}$ ray through crystal cell specimen, will transformed it into Polarized Wave $(P W)$, hence both the $P W$ and $C M F$ then interacted with the $N S M F$ as given by Eq. (17), resulted in $G S-C M F$ and $G S-P W$, leading to the result shown in Fig.4:II, but as the beam is sometimes said to be reflected from the crystal [50], and a patterns shown in Fig.6B, may be obtained by allowing an electron beam to impinged at a small glance angle, on a crystal face [8], therefore such angle will result in the shape been scattered by the electrons in atoms [21], thus GS-CMF and GS$P W$ will be reflected to the detector as pattern shown on Fig.6-C, similar to shape shown in Fig.6-D [27], depicting results obtained by Davisson and Germer [13], the intensity of $C M F$ energy is given by Eq. (4), while the energy of Polarized Wave $(P W)[1]$, is derived from Eq. (4), as

$$
B_{P W-n}=\sqrt{\frac{2 E_{R} q^{2}}{m r_{m D n}^{4} c^{2} \sin \theta}} \quad T
$$

Where, $r_{m D n}$ is the magnetic radius from center of the beam to specific radius on the $P W$, sine $\theta$ is the angle at which the x-ray or the electron $(C M F)$ is directed at the crystal as shown in Fig.6-B, and $B_{P W-n}$ is the intensity of the $P W$ when passing through the crystal. 


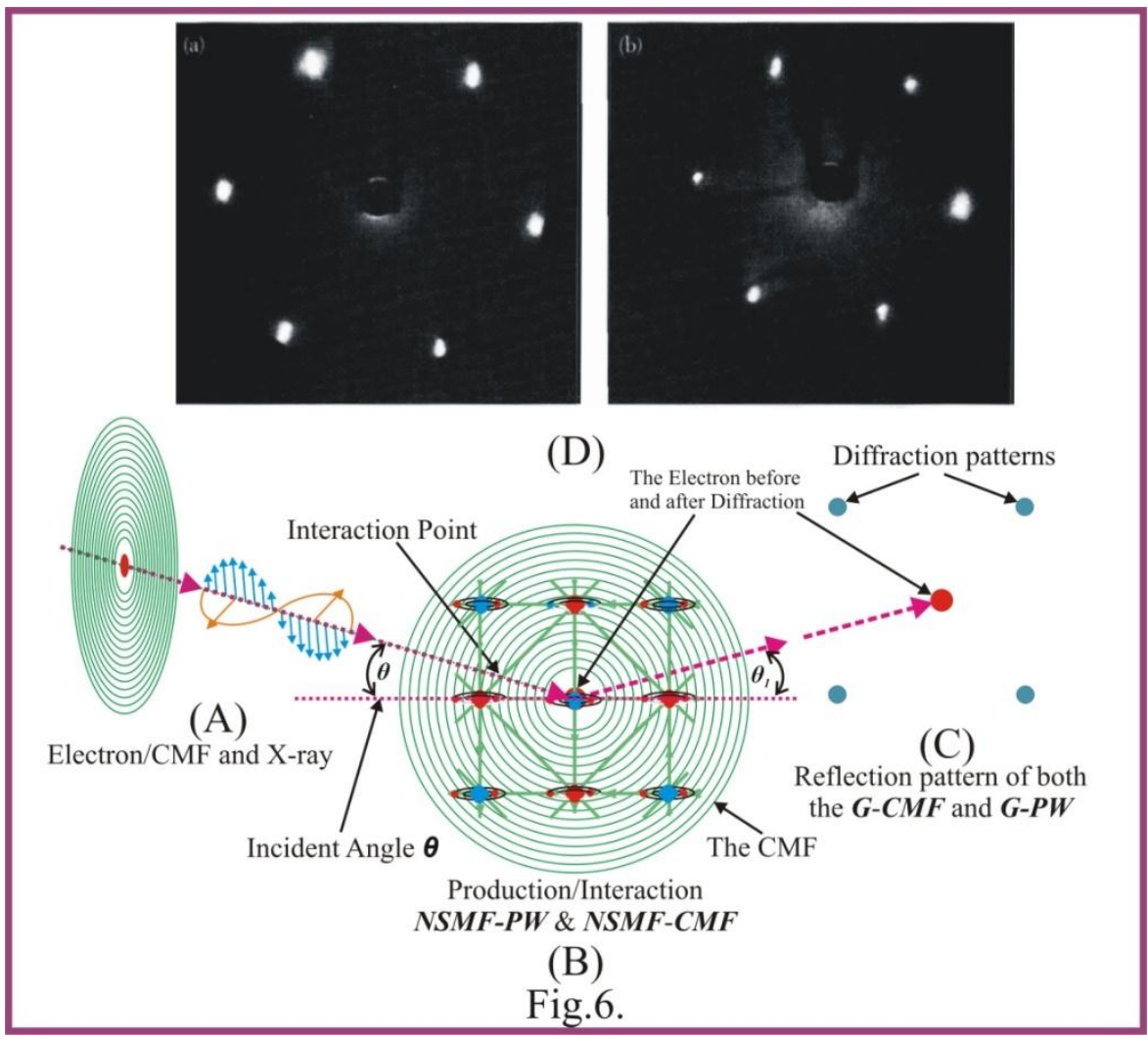

Fig.6. Both $\mathrm{x}$-ray and electron beam in (A) are directed at the crystal specimen at an angle $\theta$ in (B), the $\mathrm{x}$-ray is polarized by crystal spacing and transformed into Polarized Wave $(P W)$, similar to the Circular Magnetic Field $(C M F)$ accompanying the electron; both the $P W$ and $C M F$ interacted with the Nucleus Spinning Magnetic Field $(N S M F)$ of the crystal lattice producing dots of Glowing Spot-CMF (GS-CMF) and GS-PW, both are then deflected, imprinted and appeared as diffraction pattern on $(C)$, the electron deflected with the patterns, depicted Davisson \& Germer experiment [13] similar to patterns in (D).

And since electrons are primarily scattered by protons in the nuclei [21] similar to $\alpha$ particle experiment [6], this occurred concurrently with the modulated $G S-C M F$, which appeared on the monitor as spots patterns shown on Fig.6-C, therefore, when an electron beam or x-ray are directed at a crystal surface as shown in Fig.6A, they penetrate the specimen in designated angle $\theta$, the angle is greater at lower energy and smaller for higher energy [27], this because at smaller $\theta$ much energy is required to be obtained both the $C M F$ and $P W$ than at greater $\theta$, as shown in Fig.6-B, hence from Eq. (18), both $C M F$ and $P W$ interacted and reflected from the crystal as shown in Fig.6-B, and given by

$$
E_{C M F}=E_{P W}=\frac{B_{C M F(P W)}^{2} m r_{m D n}^{4} c^{2} \text { sine } \theta}{2 q^{2}} \quad J
$$

Where, sine $\theta$ is the angle at which both the $C M F$ and $P W$ are directed at the crystal as shown in Fig.6-B.

The phantom waves as given by Eq. (1) and Eq. (4) is the Circular Magnetic Field $(C M F)$ produced by an energetic electron, unless deflected by electric or magnetic field [23] it can't control the direction of the electron' creating it, and since the above radiation energy is from electron' energy [51], as

$$
\frac{m V_{e}^{2}}{2}=h v=\frac{h c}{\lambda}
$$

Substituting the wavelength $\lambda$ in Eq. (20) by $\lambda=4 r_{m D n}$, therefore the $C M F$ (or phantom) energy based on the radial distance is given by

$$
\frac{m V_{e}^{2}}{2}=\frac{h c}{4 r_{m D n}}
$$

From Eq. (21), the $C M F$ radius $r_{m D n}$ shown in Fig.4-II, and used in Table.3, is given by

$$
r_{m D n}=\frac{h c}{2 m V_{e}^{2}}=\frac{1.0910844279721615159355845759898 \times 10^{5}}{V_{e}^{2}}
$$


Since the $C M F$ energy on the R.H.S. of Eq. (21) depends on the magnetic radius $\left(r_{m D}\right)$, which is quarter of the wavelength [5], while the whole energy of the system depends on electron velocity $\left(V_{e}\right)$ in the L.H.S of Eq. (21), therefore the $C M F$ ' energy is proportional to electron velocity, but electron deflection is suggested to be caused by the repulsive interaction between Electron' Spinning Magnetic Field (ESMF) and Nucleus Spinning Magnetic Field (NSMF) [36]; this repulsive force is given by

$$
F_{D}=B_{E S M F} B_{N S M F} r_{r}^{2} c \sin \theta
$$

Where, $B_{E S M F}$ is the $E S M F, B_{N S M F}$ is the NSMF, $\theta$ is the angle shown in Fig.6-B, and $F_{D}$ is the deflection repulsive force; and since the depth of penetration of the $P W$ (x-ray beam) depends on the solid and on the radiation energy, typical depth is $0.01 \mathrm{~m}$, while a diffracted beam in Bragg reflection may remove the energy in a much shorter distance, perhaps $10^{-3} \mathrm{~m}$ in an ideal crystal [27], which is related to Eq. (23), hence $C M F$ is also deflected as given by Eq. (23) accompanied by the electron, therefore this is a reflection of a beam of highly-energetic electrons in a minimize penetration into a crystal by controlling the incidence angle to enhance the role of the crystal surface [2], which is synonymous to the reflection/deflection of the electron by the central body of nucleus similar to $\alpha$ particle [6], we herby suggested due to the repulsive force of both the ESMF and $N S M F$ given in Eq. (23); the reflection of electron-CMF during Davisson and Germer experiment, is occurred after an interaction between both $C M F-N S M F$ or $P W-N S M F$, leading to the formation of $G S-C M F$ and $G S-P W$, therefore the diffraction pattern in Davisson and Germer experiment showed in Fig.6-C, is a reflection of pattern created by interaction of the $C M F$ or $P W$ with the $N S M F$, or the created $G S-C M F$ and $G S-P W$.

On the other hand, substituting the wavelength $\lambda$ by $\lambda=4 r_{m D n}$ in Eq. (20), therefore the radiation energy is given by

$$
h v=\frac{h c}{4 r_{m D n}}
$$

From Eq. (24), the frequency is given by

$$
v=\frac{c}{4 r_{m D n}}
$$

Eq. (25) is equivalent to $v=\frac{c}{\lambda}$, while from Eq. (24), the radius is given in terms of energy by

$$
r_{m D n}=\frac{h c}{4 E}
$$

If the radius $4 r_{m D n}$ is replaced by $\lambda$ in Eq. (26), the de Broglie relationship is obtained.

\section{Rings' Radius and Deviation Angle}

Is there any wave produced by electron or any charged particles rather than the $C M F$ ? If not, then can other diffraction characteristics be explained differently? The Bragg angle $\theta$ was thought to be very small in electron diffraction, where electron beam is strongly diffracted from planes of atoms which are almost parallel to the electron beam, while it's very large in x-ray diffraction [15], this contradiction is reconciled by transforming the $\mathrm{x}$-ray into its $P W$ origin through the polarization carried by the slit [1], the result of which is synonymous to $C M F$ produced by an electron, hence both are treated as identical; therefore the emergence of an electron from the electron gun, encircled by the $C M F$, as shown in Fig.5-C, at which the electron' velocity started from zero at the filament, hence the $C M F$ also starts from zero, then reached maximum instantly after ejected from the cathode, thus producing maximum $C M F$ the moment electron emerged from the electron gun and entered the specimen holder in Fig.5-A\&B; and since the spots are formed by rectilinear pencils spreading in all directions from the crystal [22], and since within microscope level, the probe electrons and radiation interacted with object under study to create an image [27], as the interaction occurred at the microscopic level inside the specimen, and since diffraction is loosely defined as the flaring of light as it emerges from a narrow slit [47], and that, $C M F$ of specific intensity doesn't spread out until emerged from the crystal, therefore, the related flaring occurred at the moment when $C M F$ and $P W$ emerged from the crystal cell; although the trajectory angle of the $C M F$ started at the first crystal cell, but the line passing through each micro $C M F$ on the specimen holder formed an angle with the larger ring on the monitoring screen, can be traced back to a zero point inside the filament of the electron gun as designated by Point-x in Fig.5-C, therefore due to the flaring, the CMF's angle increased with electron's movement along the central line L, and since the movement of photographic plate backwards or forwards, shows the spots formed by rectilinear pencils spreading in all directions from the crystal [22], therefore, the $C M F$ moved and interacted with the specimen's NSMF and enlarged (amplified) by the variation of field intensity with the distance as it imprinted on the monitor screen; thus by rearranging Eq. (4), the $C M F$ ' magnetic radius $\left(r_{m D n}\right)$ inside the specimen in Fig.5-A\&B, is similar to Fig.4-II, and given by

$$
r_{m D n}=\sqrt[4]{\frac{2 E q^{2}}{B_{C M F}^{2} m c^{2}}}
$$

The magnitude of the $\boldsymbol{B}_{\boldsymbol{C M} \boldsymbol{F}}$ in terms of energy and radius is given by

DOI: $10.9790 / 4861-08050299116 \quad$ www.iosrjournals.org




$$
B_{C M F}=\sqrt{\frac{2 E q^{2}}{m c^{2} r_{m D}^{4}}}
$$

Therefore, energy required to produced specific state is

$$
\mathrm{E}=\frac{B_{C M F}^{2} m c^{2} r_{m D}^{4}}{2 q^{2}}
$$

Since electron emerged from cathode-rays in Fig.5-C surrounded by the $C M F$, therefore point-x inside cathoderays filament is where the $C M F$ magnitude started from zero, and since ring pattern originated with electron as $C M F$, hence point-x is the hypothetical point, where rings pattern's angle deviated from the central line $\mathrm{L}$ of the moving electron, this is the angle $\theta_{n}$ in Fig.5-C, it is given by

$$
\tan \theta_{n}=\frac{R_{n}}{L}
$$

Where, $R_{n}$ is the nth ring radius on the monitor screen shown in Fig.5-C as $\left(R_{1}, R_{2}\right.$ and $\left.R_{3}\right), \mathrm{L}$ is the central distance moved by an electron from cathode-rays to the monitor, $\theta_{n}$ is the angle flared (deviated) by the $C M F$ from the specimen, therefore from Eq. (30), any of the rings radiuses $\left(R_{n}\right)$ in Fig.5-C, is given by

$$
R_{n}=L \tan \theta_{n}
$$

Since $R_{n}$ is an enlarged $C M F$ radius of $r_{m D n}$ inside the crystal shown in Fig.4-II, therefore substituting $r_{m D n}$ in Eq. (27) with RHS of Eq. (31), and change L with $L_{1}$, the following is obtained

$$
L_{1}^{4} \tan \theta_{n}^{4}=\frac{2 \mathrm{E} q^{2}}{B_{C M F}^{2} m c^{2}}
$$

Where, $L_{1}$ is the distance from point-x to the specimen, hence from Eq. (32), the length $L_{1}$ in Fig.5, is given by

$$
L_{1}=\sqrt[4]{\frac{2 E q^{2}}{B_{C M F}^{2} m c^{2} \tan \theta_{n}^{4}}}
$$

The angle $\theta_{n}$ can also be given by

$$
\tan \theta_{n}=\sqrt[4]{\frac{2 \mathrm{E} q^{2}}{B_{C M F}^{2} m c^{2} L_{1}^{4}}}
$$

Therefore the $C M F\left(B_{C M F}\right)$ is given by

$$
B_{C M F}=\sqrt{\frac{2 E q^{2}}{m c^{2} L_{1}^{4} \tan \theta_{n}^{4}}}
$$

Substituting energy E in Eq. (34) with kinetic energy formula for electron, we got

$$
L_{1}^{4} \tan \theta_{n}^{4}=\frac{V^{2} q^{2}}{B_{C M F}^{2} c^{2}}
$$

Substituting $B_{C M F}$ in Eq. (1) with $B_{C M F}$ in Eq. (36), the following is derived

$$
L_{1}^{4} \tan \theta_{n}^{4}=\frac{V_{e} q r_{m D n}}{c}
$$

From Eq. (37), the length $L_{1}$ is

$$
L_{1}=\sqrt[4]{\frac{V_{e} q r_{m D n}}{c \tan \theta_{n}^{4}}}
$$

And the angle $\theta_{n}$ is

$$
\theta_{n}=\sqrt[4]{\frac{V_{e} q r_{m D n}}{c \tan L_{1}^{4}}}
$$

The typically magnitude of the produced magnetic field at point-x in Fig.5-C, varied inversely from distance $\mathrm{L}$ and angle $\theta_{n}$, and increased with potential $\mathrm{E}$; but the magnitude of the $C M F\left(B_{C M F}\right)$ produced by an electron and moving together with it from point-x to the specimen doesn't changed in magnitude, till interacted with the NSMF, and since the lens in TEM can be focused on initial image formed by the objective lens, or diffraction pattern formed in the back focal plane of the objective lens [30], therefore the role of TEM or CRT potential is to amplify the resulted changes in $C M F$ due to interaction between $C M F-N S M F$ on the monitoring screen.

Using Eq. (28) and Eq. (34), the $B_{C M F}$ and angle $\theta_{n}$ are derived and given in Table. 4, when the acceleration potential $\mathrm{E}$ increased, the radius of the two rings patterns $R_{1}$ and $R_{2}$, generated on the monitoring screen are reduced, while given the distance between the foil and the screen as $\mathrm{L}=0.135 \mathrm{~m} \mathrm{[43]}$ the table gives 
the variation of $B_{C M F}$ and angle $\theta_{n}$ with $\mathrm{E}$ and $R_{n}$; the experimenters found the relation between the rings and energy, in form of $2 D_{n} \sqrt{E}$ as constant, where $2 D_{n}$ is the diameter of the ring and $\mathrm{E}$ in electrons voltage [8], this can be tested using parameters in Table.4.

Since the $C M F$ trajectory started at the filament in the cathode-rays tube passing through the specimen, therefore the six radius shown in Fig.4-II by $r_{m D 1}$ to $r_{m D 6}$, are related to the distance $L_{1}$ from the cathode to the spacemen shown in Fig.5-C, the radius is given by

$$
r_{m D n}=L_{1} \cdot \tan \theta_{n}
$$

Where, $r_{m D n}$ is the nth radius of the $C M F$ as it interacted in the specimen with the $N S M F$, as shown in Fig.4-II, the radius is also given in Table.3; while the amplification factor, or the radius on the monitoring screen divided by the $C M F$ radius inside the crystal cell is the ratio between Eq. (31) over Eq. (40), it is

$$
A_{F}=\frac{R_{n}}{r_{m D n}}
$$

This can also be written as

$$
A_{F}=\frac{L \tan \theta_{n}}{L_{1} \cdot \tan \theta_{n}}
$$

Since $\tan \theta_{n}$ in the numerator and denominator of Eq. (42) are equal, hence the amplification is

$$
A_{F}=\frac{L}{L_{1}}
$$

Since Eq. (40) derived the inter-atomic radius, while the reflection of rings pattern on monitoring screen is derived by Eq. (31), therefore the relative radial distance of rings patterns on monitoring screen can be used to derived the radius of any group on that micro-scale, and given by

$$
\frac{r_{m D 1}}{r_{m D n}}=\frac{R_{1}}{R_{2}}
$$

\begin{tabular}{|c|c|c|c|c|c|c|}
\hline E-kV & $R_{1} \mathrm{~m}$ & $B_{C M F} \mathrm{~T}$ & $\theta_{n}$ & $R_{2} \mathrm{~m}$ & $B_{C M F} \mathrm{~T}$ & $\theta_{n}$ \\
\hline 3.0 & 0.0165 & $\begin{array}{r}6.37246875630898562 \\
4544276776905 \times 10^{-17}\end{array}$ & $\begin{array}{l}6.96825674137854354 \\
048351229576560\end{array}$ & 0.02625 & $\begin{array}{l}2.5177754106559584006 \\
399534661242 \times 10^{-17}\end{array}$ & $\begin{array}{c}11.00354085174950323 \\
7566329686443 \mathrm{o}\end{array}$ \\
\hline 3.5 & 0.01415 & $\begin{array}{c}9.359143762226355 \times \\
10^{-17}\end{array}$ & $\begin{array}{l}5.98359812110036182 \\
97982679803818 \mathrm{o}\end{array}$ & 0.0244 & $\begin{array}{l}3.1475261386931712571 \\
08012288679 \times 10^{-17}\end{array}$ & $\begin{array}{c}10.24507778056125467 \\
9997576153452 \mathrm{o}\end{array}$ \\
\hline 4.0 & 0.0133 & $\begin{array}{c}1.1325090720144688 \\
\times 10^{-16}\end{array}$ & $\begin{array}{l}5.62653865439296861 \\
72822598372474 \mathrm{o}\end{array}$ & 0.0229 & $\begin{array}{l}3.8200936242375122640 \\
865701217983 \times 10^{-17}\end{array}$ & $\begin{array}{c}9.627419446890186097 \\
6820906467037 \mathrm{o}\end{array}$ \\
\hline 4.5 & 0.012 & $\begin{array}{c}1.4755663433039074 \\
\times 10^{-16}\end{array}$ & $\begin{array}{l}5.07960786001456993 \\
82326653597147 \mathrm{o}\end{array}$ & 0.02175 & $\begin{array}{l}4.4916169308656396167 \\
692311548076 \times 10^{-17}\end{array}$ & $\begin{array}{c}9.152339049401993469 \\
3399425713332 \mathrm{o}\end{array}$ \\
\hline 5.0 & 0.01165 & $\begin{array}{c}1.6502438667815727 \\
\times 10^{-16}\end{array}$ & $\begin{array}{l}4.93219435110401088 \\
55500717204566 \mathrm{o}\end{array}$ & 0.0206 & $\begin{array}{l}5.2779532286092470217 \\
178567940621 \times 10^{-17}\end{array}$ & $\begin{array}{c}8.675985972667117165 \\
7618334882197 \mathrm{o}\end{array}$ \\
\hline
\end{tabular}

Therefore, the unknown $r_{m D n}$ of the specimen is

$$
r_{m D n}=\frac{R_{2} r_{m D 1}}{R_{1}}
$$

Eq. (31) is used to drive $R_{n}$ in Table.3, while Eq. (8) is used to derive $r_{m D n}$.

Table. 4. Given is the accelerating potential E, the first and second generated rings patterns radius $\left(R_{1}\right.$ and $R_{2}$ ) on the monitoring screen, distance between graphite foil and screen $\mathrm{L}=0.135 \mathrm{~m}$ [43], the $C M F$ and the angle $\theta_{n}$ for both radius are derived using Eq. (28) and Eq. (34) respectively.

\section{Results and discussion}

As given by Eq. (1), the $C M F$ produced by an accelerated electron, is also produced by fast moving nuclei with electric charges in Relativistic Heavy Ion Collider (RHIC) and Large Hadrons Collider (LHC) creating extreme strongest magnetic fields [1,52], for electron, $C M F$ is the main energy of the Electromagnetic Radiation $(E M-W)[51]$, therefore the so-called phase waves or matter waves or pilot wave which exhibit certain striking similarity with electromagnetic waves, particularly in their ability to produce the diffraction effects [5], is just the $C M F$.

An accelerated electron produced Circular Magnetic Field $(C M F)$, the magnitude of which at radial distance is related to the accelerated potential as given by Eqs. $(2,4,27 \& 35)$, therefore both Davisson an Germer experiment [13], and Thomson experiment [15], are interpreted as an interaction between the $C M F$ and the Nucleus Spinning Magnetic Field (NSMF).

A model of crystal is suggested, in which atoms are jointed by the Spinning Magnetic Force $(S M F c)$ created by the Spinning Magnetic Field $(S M F)$ produced by atom nucleus.

Since $\mathrm{x}$-ray is an Electromagnetic Radiation $(E M-R)$, hence when it passed through the crystal it will be polarized, and represented the well known Conical Diffraction $(C D)$ beam [4], and designated as the Polarized Wave (PW) [1]. 
From the above point, an Electromagnetic Radiation $(E M-R)$ with very small wavelength, is polarized by the small crystal lattice, removing its electric field, the wave retained its magnetic part in the form of the Polarized Wave $(P W)$.

Since NSMF is produced by spinning nucleus and both $C M F$ and $P W$ are produced by accelerated electron and polarized x-ray respectively, therefore both electron' $C M F$ and $P W$ from EM-R interacted with four Nucleus Spinning Magnetic Field (NSMF) at crystal corners, transformed the invisible $C M F$ or $P W$ into Glowing Spot Circular Magnetic Field $(G S-C M F)$ and Glowing Spot Polarized Wave $(G S-P W)$, at the monitoring device.

As the Polarized Wave $(P W)$ [1] originated from the electron's $C M F$ during the Flip-Flop $(F-F)$ mechanism that produced $E M-R$ [5], hence both the $C M F$ and $P W$ are identical in structure and characteristics, as it composed of an intense magnetic field, therefore this explained the resulted ring patters produced by electron wave $(C M F)$ and $E M-R$ diffracted as $P W$.

Electron/x-rays diffraction is an interaction of $C M F / P W$ with crystal, and they are divided into two parts:

a) The deflection of the electron with its $C M F$, and reflection of $P W$ after polarized from x-rays, takes place after both interacted with the Nucleus Spinning Magnetic Field (NSMF), and resulted in the Glowing SpotCMF $(G S-C M F)$ and Glowing Spot-PW $(G S-P W)$ patterns, such as Davisson and Germer Experiment.

b) The continuous flow of electrons surrounded by $C M F$ through crystal, interacted with the NSMF, producing the $G S-C M F$, while the continuous flow of $P W$ transformed from an x-ray, interacted with crystal's $N S M F$, producing an amplified the $G S-P W$ on the screen.

The magnitude of intensity and radius of produced ring patterns is a representation of resulted interaction between $C M F-N S M F$ and $P W-N S M F$, which in turn proportional to the accelerating potential for $C M F$ and wave intensity for $P W$.

The interaction of $C M F$ with the Spinning Magnetic Lines of Force $(S M L F)$ produced the Kikuchi lines; therefore, the Kikuchi lines represents the bonds which joined atoms to form the crystal structure, in other word; Kikuchi lines are lines of force connecting NSMF poles.

As X-ray diffraction determined the precise location of all atoms or molecules in the unit cell [53], this is due to the fisheye eyes lens nature of $G S-C M F$ and $G S-P W$ photographic result of the interaction, and the resulted interaction is much intense when the $C M F$ interacted with NSMF.

As electron interacted with matter about $10^{4}$ stronger than x-Rays? [39], this is because the energy of $P W$ polarized from x-ray is less intense than the $C M F$ created by moving electron, and since electrons diameter is $2 \times 10^{-15} \mathrm{~m}$, while diameter of atom is around $10^{-10}$ meters, therefore an energetic electrons are moving in a vast distance $\left(>>10^{-10} \div 2 \times 10^{-15}\right)$ between atoms or 50,000 times electrons diameter and for such a distance as shown in Fig.4-II, the strength of interaction between electron's $C M F$ with Nucleus Spinning Magnetic Fields $(N S M F)$ depends on electron' velocity, and magnitudes of $C M F$ within this distance as given by Eq. (1), which is very intense, hence resulted in more strong interaction than the PW.

The mechanism that produced $G-C M F$ and $G-P W$, can be related to photos of sunspots magnetic lines of force seen as curl lines in extreme ultraviolet wavelength [37].

Different parameters such as Ring Pattern Radius $\left(R_{n}\right)$, Deflection Angle $(\theta)$, Distance Between Cathode Rays and Monitoring Screen $(L)$ are derived similar in nature to the diffraction.

\section{Conclusion}

The diffraction phenomenon has been interpreted as a change in the characteristics of $E M-R$, that is when an EM-R passed through a small slit/crystal, which polarized the radiation, in that process the electric part of the field is removed, hence transforming the $E$ - $M R$ into Circular Magnetic Field $(C M F)$, the $C M F$ is not semicircular, rather it is a full circular wave, and composed of the magnetic part of the $E M-R$, originated from accelerated electrons [5], the wave neither travel with speed of light, nor carrying electric field, this resulted wave is what had been known as the Conical Diffraction $(C D)$ beam [4], it is designated as the Polarized Wave $(P W)$, therefore the passing of $\mathrm{x}$-ray through the crystal, resulted in $P W$, which is similar to the passing of $E M-R$ through a hole/slit/crystal [17].

A model for such crystal is suggested in which atoms are bonded by the Spinning Magnetic Force $(S M F s)$, created by Spinning Magnetic Field $(S M F)$, bonding atoms together to form crystal cell; hence, the passing of x-rays through crystal, resemble the passing of $E M-R$ through a slit/hole, thus the diffraction removed the electric field from the $\mathrm{x}$-ray, and resulted in the $P W$, which is alike the $C M F$ produced by an accelerated electron entering the crystal; therefore the existence of an intense $C M F$ in crystals due to an accelerated electron and $P W$ from polarized x-ray, both interacted with the Nucleus Spinning Magnetic Field (NSMF) produced by atoms in crystals [36], resulted in Glowing Spot-CMF (GS-CMF) and Glowing Spot-PW (GS-PW), both appears on the monitoring screen as dots/rings or reflected as dots patterns on detector when the X-ray or the electrons beams entered at a small angle, the Spinning Magnetic Lines of Force (SMLF) extended from the NSMF to form 
the crystal bond, it interacted with the $C M F$ and $P W$ to produced a Glowing NSMF-CMF (G-NSMF-CMF) and Glowing NSMF-PW (G-NSMF-PW), showed as Kikuchi lines.

As the accelerated electron surrounded by the $C M F$, moved from cathode rays, through the specimen, to the screen where the $C M F$ formed rings patterns, the angle $\theta$ between electron trajectory and the $C M F$ on which $G S$-CMF imprinted on the screen and formed the rings radius are derived, as well as this radius and the line $(L)$ moved by the electron from cathode to the screen.

From these, it's clear that, the relationship between Thomson experiment using electrons and HullDebye-Scherrer experiment using x-rays is the Circular Magnetic Field $(C M F)$ and the Polarized Wave $(P W)$, thus both are identical, and gives similar results, given by the resulted diffraction, therefore, it is clear that, G.P. Thomson was correct in realizing de Broglie's theory as a theory of light and electronic orbits, not as a theory of electron diffraction [23].

\section{Acknowledgment}

This work in particular and the previous are not imaginable without a blessing from the Almighty God, to whom I expressed my humble gratitude for been selected and empowered with such immense knowledge and insight.

\section{Reference}

[1]. M E Yousif, The Double Slit Experiment Re-Explained. IOSR J. of Appl Phys (IOSR-JAP), e-ISSN: 2278-4861.Volume 8, Issue 4 Ver. III, PP86-98, DOI: 10.9790/4861-0804038698, 2016.

[2]. D Cassidy, G. Holton, J. Rutherford, Understanding Physics, Springer-Verlag, New York, 2002.

[3]. B Crowell, Light and Matter, Benjamin Crowell, 1998.

[4]. M. V. BERRY, M. R. JEFFREY, J. G. LUNNEY, Conical diffraction: observations and theory, Proceeding of the Royal Society, doi:10.1098/rspa.2006.1680, Proc. R. Soc. A 462, 1629-1642, 2006.

[5]. M E Yousif, The Electromagnetic Radiation Mechanism, International Journal of Fundamental Physical Sciences (IJFPS), vol. 4, 2014a.

[6]. Harnwell, Livinggood Experimental Atomic Physics, McGraw-Hill, 1961.

[7]. L. L. Williams, The Spirit of Reason, Online Edition, KONFLUENCE PRESS Manitou Springs, Colorado, 2006.

[8]. R. Beeching, Electron Diffraction, Methuen \& Co. Ltd. 36 Essex Street, W.C. London, 1936.

[9]. L. de Broglie, The wave nature of the electron Nobel Lecture, 1929.

[10]. A Einstein \& into English T, Concerning an Heuristic point of view toward the emission and transformation of light, American Journal of Physics, vol. 33, 1965.

[11]. A H Compton, "A Quantum Theory of the Scattering of X-Rays by Light Elements", Physical Review, vol. 21, 1923.

[12]. L. Marion, H. M. Van, Electron Diffraction, Encyclopedia of Physics, Ed Lerner, R. G. and Trigg, G. L., VCH Publishers, Inc., 303$305,1991$.

[13]. C J Davisson, L. Germer H, Diffraction of Electrons by a Crystal of Nickel, Phys. Rev. 30, 705, 1927.

[14]. Advanced Physics Laboratory, Electron Diffraction and Crystal structure, University of Michigan, 2006.

[15]. G. P. Thomson (1928) Proc. Roy. Soc. 117, 600.

[16]. L N Hoang, The Essence of Quantum Mechanics, Science 4 All, 2013.

[17]. M E Yousif, The Compton Effect Re-Visited, J Adv Appl Phys, 1:004, 2016.

[18]. J B Hastings, F. M. Rudakov, D. H. Dowell, J. F. Schmerge, J. D. Cardoza, J. M. Castro, S. M. Gierman, H. Loos, P. M. Weber, ULTRAFAST TIME-RESOLVE D ELECTRON DIFFRACTION WITH MEGAVOLT ELECTRON BEAMS, Applied Physics Letters, SLAC-PUB-12162, 2006.

[19]. Electron Diffraction, In-class group activity 5. http://electron6.phys.utk.edu/phys250/modules/module\%202/electron_diffraction.htm

[20]. W. Clegg X-Ray Crystallography, Oxford University Press, United Kingdom, ISBN 10: 0198700970 ISBN 13: 9780198700975 , 2015.

[21]. C. Hammond, The basics of crystallography and diffraction (third edirion), Institute for Materials Research, University of Leeds, INTERNATIONAL UNION OF CRYSTALLOGRAPHY, Oxford University Press, 2009.

[22]. W. L. BRAGG, The Diffraction of Short Electromagnetic Waves by a Crystal, Proc. Camb. Phil. Soc., XVII (I), 1912.

[23]. J. Navarro, Planck and de Broglie in the Thomson Family, Christian Joas, Christoph Lehner, and Jürgen Renn (eds.), Conference on the History of Quantum Physics, Max Planck Institute for the History of Science, 2008.

[24]. M. Alonso, E. J. Finn, Fundamental University Physics V. II Field and Waves, Addison and Wesley, Massachusetts, 1967.

[25]. J. R. Ballif, Conceptual Physics, Wiley N. Y., 1969.

[26]. W. R. Fuch, Modern Physics Weidenfield \& Nicolson (Educational) Ltd: and The Macmillan for Translation, Zurich, 1967.

[27]. C. Kittel, Introduction to Solid-State Physics, John Wiley \& Sons, Inc, 1957.

[28]. M. M. Ripoll, "Crystallography-Cristalografia", Dept. of Crystallography \& Struc. Spanish National Research Council, Madrid, 2016.

[29]. M E Yousif, The Magnetic Interaction, Comprehensive Theory Articles, Journal of Theoretics, vol. 5, 2003a.

[30]. University of Nevada, Basic Crystallography and Electron Diffraction from Crystals, CHEM 793, 2008 Fall Chapter 3, Lecture 14 Las Vegas, 2012.

[31]. MSE 2090, Introduction to the Science and Engineering of Materials, University of Virginia, Department of Materials Science and Engineering, 2010.

[32]. Wikipedia, Kikuchi Line, From Wikipedia, the free encyclopedia, 2016.

[33]. J. J. Fundenberger, A. Morawiec, E. Bouzy, J. S. Lecomte, Polycrystal orientation maps from TEM, Ultramicroscopy, Volume 96, Issue 2, 127-137, 2003.

[34]. W. Qin, P. Fraundorf, Lattice fringe visibility after tilt, arXiv:physics.optics/00mmxxx in prep.

[35]. M. V. Gomoyunova, I. I. Pronin, N. S. Faradzhev, D. A. Valda tsev, Kikuchi-band formation in medium-energy electron-diffraction patterns, PHYSICS OF THE SOLID STATE, Vo. 41, No 3, 1999.

[36]. M E Yousif, THE SPINNING MAGNETIC FORCE, Comprehensive Theory Articles, Journal of Theoretics, vol. 5, $2003 \mathrm{~b}$. 
[37]. Courtesy of SOHO/EIT consortium. SOHO is a project of international cooperation between ESA and NASA. http://sohowww.nascom.nasa.gov/hotshots/2005_05_20/figure_2.jpg

[38]. G. J. C. Carpenter, J. F. Watters ELECTRON DIFFRACTION PATTERNS FOR H.C.P. a-ZIRCONIUM, Chalk River Nuclear Laboratories Chalk River, Ontario, 1972.

[39]. S. Nicolopoulos, D. Bultreys, Consultant IUCr Electron Crystallography Commission, "Precession electron diffraction and TEM applications." NanoMEGAS SPRL, Blvd Edmond Machtens 79, B-1080 Brussels, Belgium.

[40]. B. M. Lynne, C. Baerlocher, Electron crystallography as a complement to X-ray powder diffraction techniques, Z. Kristallogr, 228,1-10/DOI 10.1524/zkri.2013.1558, Oldenbourg Wissenschaftsverlag, Munchen, 2013.

[41]. S. Ino, D. Watanabe, S. Ogawa, Epitaxial Growth of Metals on Rock Salt faces Cloven in Vacuum, J. Phys. Soc. Japan 17, 1074$1075,1962$.

[42]. B. M. Moskowitz, Hitchhiker's Guide to Magnetism, the Environmental Magnetism Workshop held 1991 at the Institute for Rock Magnetism, 1991.

[43]. LD Physics leaflets, Atomic and Nuclear Physics Introductory experiments Dualism of wave and particle, LD Didactic GmbH Leyboldstrasse 1 D-50354 Huerth / Germany.

[44]. B. H. J. Fultz, Transmission Electron Microscopy and Diffractometry of Materials, Graduate Texts in Physics, DOI 10.1007/978-3642-29761-8_1, Springer-Verlag Berlin Heidelberg, Chapter 1, Diffraction and the X-Ray Powder Diffractometer, 2013.

[45]. M. Blackman, On the Intensities of Electron Diffraction Rings, Proceedings of the Royal Society of London. Series A, Mathematical and Physical Sciences, Vol. 173, No. 952, pp. 68-82, 1939.

[46]. D. GABOR, THE ELECTRON MICROSCOPE Its Development, Present Performance and Future Possibilities, CHEMICAL PUBLISHINC CO., INC., Brooklyn, New York, 1948.

[47]. Physics 50 Webpage, 37 Diffraction, Spring, 2010.

[48]. R. F. W. Bader, An Introduction to the Electronic Structure of Atoms and Molecules, Chapter 5, Section 1.

[49]. Wikipedia, Atomic radii of the elements (data page), From Wikipedia, the free encyclopedia, 2016b.

[50]. H. Semat, K. Robert, "Physics, Chapter 43: X-Rays". Robert Katz Publications, 1958.

[51]. M E Yousif, Electromagnetic Radiation Energy and Planck' Constant, International Journal of Innovative Research in Advanced Engineering (IJIRAE), vol. 1, 2014b.

[52]. K. Itakura, Strong Field Dynamics in Heavy Ion Collisions, K. Itakura, et al., (edited) "Proceedings of International Conference on Physics in Intense Fields (PIF2010)," edited by K. Itakura, et al, 2010b.

[53]. O. Grubor-Urosevic, "X-ray diffraction study of dysprosium single crystal samples in a Diamond Anvil Cell (DAC)", 2009. 